\title{
A MINIMAX FORMULA FOR DUAL $B^{*}$-ALGEBRAS
}

\author{
BY
}

\section{PAK-KEN WONG}

\begin{abstract}
Let $A$ be a dual $B^{*}$-algebra. We give a minimax formula for the positive elements in $A$. By using this formula and some of its consequent results, we introduce and study the symmetric norms and symmetrically-normed ideals in $A$.
\end{abstract}

1. Introduction. Let $H$ be a (complex) Hilbert space and $L C(H)$ the algebra of all compact operators on $H$. Then $L C(H)$ is a simple dual $B^{*}$-algebra and every simple dual $B^{*}$-algebra is of this form. The minimax formula for the positive elements in $L C(H)$ is well known and has many applications (see $[2$, p. 908, Theorem 3] and [3, p. 25, Theorem]). In this paper, we present a generalization of this formula to the positive elements in an arbitrary dual $B^{*}$-algebra. Let $a$ be a positive element in a dual $B^{*}$-algebra $A$ and $E$ the set of all Hermitian minimal idempotents in $A$. We show that the singular values $s_{n}(a)$ of $a$ can be calculated by the following equations

$$
\begin{aligned}
s_{1}(a) & =\max \{\| \text { eae } \|: e \in E\}, \\
s_{n+1}(a) & =\min _{f_{1}, \ldots, f_{n} \in E} \max \left\{\| \text { eae } \|: e \in E, e f_{i}=0, i=1,2, \ldots, n\right\} .
\end{aligned}
$$

After establishing this formula, we give some applications. Let $R_{0}=(0)$ and let $R_{n}$ be the set of all elements $x=\sum_{j=1}^{n} x_{j} f_{j}$ in $A$, where $x_{j} \in A$ and $f_{j} \in E$ such that $f_{i} f_{j}=0(i \neq j)$. We show that, for any element $a$ in $A$, its singular values are given by

$$
s_{n+1}(a)=\min \left\{\|a-b\|: b \in R_{n}\right\} \quad(n=0,1,2, \ldots) .
$$

We also obtain that, for all elements $a, b$ in $A$,

$$
\sum_{n=1}^{k} s_{n}(a+b) \leqslant \sum_{n=1}^{k} s_{n}(a)+\sum_{n=1}^{k} s_{n}(b) \quad(k=1,2, \ldots)
$$

and

Received by the editors October $1,1975$.

AMS (MOS) subject classifications (1970). Primary 46C05; Secondary 46K99.

Key words and phrases. Dual algebra, $A^{*}$-algebra, $B^{*}$-algebra, Hermitian minimal idempotent, symmetrically-normed ideal, symmetric norming function.

Copyright 0 1977, American Mathematical Society 


$$
\prod_{n=1}^{k} s_{n}(a b) \leqslant \prod_{n=1}^{k} s_{n}(a) \prod_{n=1}^{k} s_{n}(b) \quad(k=1,2, \ldots)
$$

These inequalities were obtained by K. Fan and A. Horn for compact operators on a Hilbert space (see [3, p. 48, Lemma 4.2]).

The properties of symmetric norming functions and symmetric norm (uniform crossnorm) in $L C(H)$ are well known and have been studied by many mathematicians (e.g. see [3] and [8]). In this paper, we introduce the concepts of symmetric norm and symmetrically-normed ideals for $A$. Let $S_{A}$ be the socle of $A$. We show that the class of all s.n. functions and the class of all symmetric norms on $S_{A}$ generate each other.

Let $\Phi$ be an s.n. function, $A_{\Phi}$ the s.n. ideal generated by $\Phi$ and $A_{\Phi}^{(0)}$ the closure of $S_{A}$ in $A_{\Phi}$. We prove that $A_{\Phi}^{(0)}$ is a dual $A^{*}$-algebra and the conjugate space of $A_{\Phi}^{(0)}$ can be identified with $A_{\Phi^{*}}$, where $\Phi^{*}$ denotes the s.n. function adjoint to $\Phi$. The formulas established above are useful in the proof of these results. We also remark that for the case $A=L C(H)$, all these results were known.

In this paper, our approach is elementary and basically algebraic. The technique in the proof of the minimax formula is quite different from that used in [2] and [3].

2. Notation and preliminaries. Definitions not explicitly given are taken from Rickart's book [6].

For any set $S$ in a Banach algebra $A$, let $l_{A}(S)$ and $r_{A}(S)$ denote the left and right annihilators of $S$ in $A$, respectively. Then $A$ is called a dual algebra, if for every closed right ideal $R$ and every closed left ideal $I$, we have $r_{A}\left(l_{A}(R)\right)=$ $R$ and $l_{A}\left(r_{A}(I)\right)=I$. See [5] and [6] for some of its properties.

An idempotent $e$ in a Banach algebra $A$ is said to be minimal if $e A e$ is a division algebra. In case $A$ is semisimple, this is equivalent to saying that $A e(e A)$ is a minimal left (right) ideal of $A$.

Let $A$ be a Banach algebra. A bounded linear operator $T$ on $A$ is called a right centralizer if $T(x y)=(T x) y$ for all $x, y$ in $A$. For each $a$ in $A$, the operator $L_{a}: x \rightarrow a x(x \in A)$ is a right centralizer on $A$.

In this paper, all algebras and linear spaces under consideration are over the field $C$ of complex numbers.

Notation. In this paper, $A$ will denote a dual $B^{*}$-algebra with norm $\|\cdot\|$.

We shall use, without explicitly mentioning, the following fact: For any orthogonal family $\left\{e_{\alpha}\right\}$ of Hermitian minimal idempotents of $A, \Sigma_{\alpha} e_{\alpha} x$ is summable in $A$, and especially when $\left\{e_{\alpha}\right\}$ is a maximal family, $x=\Sigma_{\alpha} e_{\alpha} x$ for all $x$ in $A$ (see [5, p. 30, Theorem 16] and [10, p. 442, Theorem 5.2]).

Let $b$ be a normal element in $A$ and $\operatorname{Sp}_{A}(b)$ the spectrum of $b$ in $A$. Then 
it is well known that $\mathrm{Sp}_{A}(b)$ is either finite or countable, and has no nonzero limit points (see [1, p. 502, Corollary]). Let $\left\{e_{\alpha}\right\}$ be a maximal orthogonal family of Hermitian minimal idempotents in $A$ such that $e_{\alpha} b=b e_{\alpha}$ for all $\alpha$. By [6, p. 111, Theorem (3.1.6)], each $\lambda_{\alpha} \in \operatorname{Sp}_{A}(b)$, where $\lambda_{\alpha} e_{\alpha}=b e_{\alpha}=e_{\alpha} b e_{\alpha}$, and $\operatorname{Sp}_{A}(b)-(0) \subset\left\{\lambda_{\alpha}\right\}$. Let $\lambda$ be a nonzero number in $\operatorname{Sp}_{A}(b)$. If the set $\left\{\lambda_{\alpha}: \lambda_{\alpha}=\lambda\right\}$ has $k_{\lambda}$ elements, then the number $k_{\lambda}$ is finite and independent of the choice of $\left\{e_{\alpha}\right\}$ (see the proof of Lemma 2.3 in [11]). We call $k_{\lambda}$ the multiplicity of $\lambda$. Let $\left\{\lambda_{n}\right\}=\left\{\lambda_{\alpha}: \lambda_{\alpha} \neq 0\right\}$. Then $\left\{\lambda_{n}\right\}$ is countable and $b=$ $\Sigma_{\alpha} e_{\alpha} b=\Sigma_{n} \lambda_{n} e_{n}$, where $e_{n} \in\left\{e_{\alpha}\right\}$ with $e_{n} b=\lambda_{n} e_{n}$ (see [11]). It is clear that $\left\{\lambda_{n}\right\}$ is independent of $\left\{e_{\alpha}\right\}$ and, if $\lambda_{\alpha} \neq \lambda_{n}$ for all $n$, then $\lambda_{\alpha}=0$. The numbers $\lambda_{n}$ are called the eigenvalues of $b$.

Now suppose $a$ is a nonzero element in $A$. Then $a^{*} a$ is a positive element and so each nonzero number in $\operatorname{Sp}_{A}\left(a^{*} a\right)$ is positive. Let $\left\{\lambda_{n}\right\}$ be the eigenvalues of $a^{*} a$, arranged in decreasing order and repeated according to multiplicity. Then $\lambda_{n} \geqslant 0$ and $\lambda_{n} \rightarrow 0$ as $n \rightarrow \infty$. Let $\left\{e_{\alpha}\right\}$ be a maximal orthogonal family of Hermitian minimal idempotents of $A$ such that $e_{\alpha} a^{*} a=a^{*} a e_{\alpha}$ for all $\alpha$. Then $a^{*} a=\Sigma_{\alpha} a^{*} a e_{\alpha}=\Sigma_{n} \lambda_{n} e_{n}$ and

$$
a=\sum_{n} a e_{n}
$$

where $e_{n} \in\left\{e_{\alpha}\right\}$ with $\lambda_{n} e_{n}=a^{*} a e_{n}$ (see [11]). Put $s_{n}(a)=\sqrt{\lambda_{n}}$.

DEFinition. The number $s_{n}(a)$ is called the $n$th singular value of the element $a$ in $A$.

REMARK. Since $\lambda_{1}=\left\|a^{*} a\right\|=\|a\|^{2}, s_{1}(a)=\|a\|$.

Let us put

$$
[a]=\sum_{n} s_{n}(a) e_{n} .
$$

Then $[a]=[a]^{*}=\left(a^{*} a\right)^{1 / 2}$ (see [11]). Define two mappings $W$ and $W^{*}$ on $A$ into itself by

$$
W x=\sum_{n}\left(s_{n}(a)\right)^{-1} a e_{n} x \quad(x \in A)
$$

and

$$
W^{*} x=\sum_{n}\left(s_{n}(a)\right)^{-1} e_{n} a^{*} x \quad(x \in A) .
$$

Then we can show that $W$ and $W^{*}$ are right centralizers on $A$ with $\|W\|=\left\|W^{*}\right\|$ $=1, W[a]=a$ and $W^{*} a=[a]$ (see [11]). We shall refer to the operator $W$ as the partial isometry asssociated with $a$.

3. A minimax formula for $A$. Let $A$ be a dual $B^{*}$-algebra with norm $\|\cdot\|$ and $E$ the set of all Hermitian minimal idempotents in $A$. Then by $[6$, p. 98 , 
Lemma (2.8.6)] and [6, p. 261, Lemma (4.10.1)], every nonzero left or right ideal of $A$ contains some element of $E$.

LEMMA 3.1. Let $M$ be a maximal modular right ideal and $R$ a nonzero right ideal of $A$ such that $M \cap R=(0)$. Then $R$ is a minimal right ideal of $A$.

Proof. By [6, p. 98, Lemma (2.8.6)], $R$ contains a minimal right ideal $I$. Since $M$ is maximal, it follows that $M \oplus R=M \oplus I=A$. Therefore $R=I$.

LEMMA 3.2. Let $M_{1}, M_{2}, \ldots, M_{n}$ be maximal modular right ideals and $e_{1}$, $e_{2}, \ldots, e_{n+1}$ any mutually orthogonal Hermitian minimal idempotents in $A$. Then

$$
M_{1} \cap M_{2} \cap \cdots \cap M_{n} \cap\left(e_{1}+e_{2}+\cdots+e_{n+1}\right) A \neq(0) .
$$

PROOF. We use induction. If $k=1$, the lemma follows easily from Lemma 3.1. Now suppose that the lemma is true for $k=n-1$. Since

$$
\begin{aligned}
& \left(e_{m+1}+e_{m+2}+\cdots+e_{m+p}\right) \\
& \quad=\left(e_{1}+e_{2}+\cdots+e_{n+1}\right)\left(e_{m+1}+e_{m+2}+\cdots+e_{m+p}\right)
\end{aligned}
$$

with $1 \leqslant m+p \leqslant n+1$, we see that $\left(e_{m+1}+e_{m+2}+\cdots+e_{m+p}\right) A \subset$ $\left(e_{1}+e_{2}+\cdots+e_{n+1}\right) A$. If there exists some $e_{i} \in M_{1} \cap M_{2} \cap \cdots \cap M_{n}$ $(1 \leqslant i \leqslant n)$, then (3.1) clearly holds. Therefore, without loss of generality, we may assume that $e_{1} \notin M_{1}$. It follows from Lemma 3.1 that $M_{1} \cap\left(e_{1}+e_{j}\right) A$ $\neq 0(j=2,3, \ldots, n+1)$. Hence for each $j$, there exists a Hermitian minimal idempotent $h_{j} \in M_{1} \cap\left(e_{1}+e_{j}\right) A$; clearly $h_{j}=\left(e_{1}+e_{j}\right) h_{j}$. We claim that $e_{j} h_{j}$ $\neq 0$. In fact, if $e_{j} h_{j}=0$, then $h_{j}=e_{1} h_{j} \in e_{1} A$. Therefore, by [6, p. 261, Lemma (4.10.1)], $e_{1}=h_{j} \in M_{1}$; a contradiction. Hence $e_{j} h_{j} \neq 0$. If there exists some $2 \leqslant p \leqslant n+1$ such that $h_{p} A \cap \Sigma_{j \neq p} h_{j} A \neq(0)$, then $h_{p} \in h_{p} A \subset$ $\Sigma_{j \neq p} h_{j} A$. Hence $h_{p}=\Sigma_{j \neq p} h_{j} x_{j}$ with $x_{j} \in A$. Then $e_{p} h_{p}=$ $\Sigma_{j \neq p} e_{p}\left(e_{1}+e_{j}\right) h_{j} x_{j}=0$, which is a contradiction. Consequently, $h_{2} A+h_{3} A$ $+\cdots+h_{n+1} A$ is a direct sum. Therefore by the proof of $[1$, p. 497, Theorem 2.2], we can find an orthogonal family $\left\{f_{2}, f_{3}, \ldots, f_{n+1}\right\}$ of Hermitian minimal idempotents contained in $h_{2} A+h_{3} A+\cdots+h_{n+1} A$. Hence by induction hypothesis, $M_{2} \cap M_{3} \cap \cdots \cap M_{n} \cap\left(f_{2}+f_{3}+\cdots+f_{n+1}\right) A \neq(0)$. Since by (3.2), $h_{j} \in\left(e_{1}+e_{j}\right) A \subset\left(e_{1}+e_{2}+\cdots+e_{n+1}\right) A$, it follows easily that $\left(f_{2}+f_{3}+\cdots+f_{n+1}\right) A \subset M_{1} \cap\left(e_{1}+e_{2}+\cdots+e_{n+1}\right) A$, and so (3.1) holds. This completes the proof.

LEMma 3.3. Let a be a positive element and e any Hermitian minimal idempotent in $A$. Then

(i) eae is positive.

(ii) If $\lambda e=e a e$, then $\lambda=\|e a e\|$. 
Proof. (i). Write $a=h^{*} h$, with $h \in A$. Then eae $=(h e)^{*}(h e)$ is positive. (ii). It follows easily from $[6$, p. 261 , Theorem (4.10.3)] that $\lambda \geqslant 0$. Therefore $\lambda=\|$ eae $\|$.

We now have the main result of this section.

THEOREM 3.4 (MINIMAX FORMULA). Let a be a positive element in a dual $B^{*}$-algebra $A$ with singular values $\left\{s_{1}(a), s_{2}(a), \ldots\right\}$ and $E$ the set of all Hermitian minimal idempotents in $A$. Then for $n=1,2, \ldots$ we have

$$
\begin{aligned}
& s_{1}(a)=\max \{\|e a e\|: e \in E\} \\
& s_{1}(a)=\min _{f_{1} \in E} \max \left\{\|e a e\|: e \in E, f_{1} e=0\right\} \\
& : \\
& s_{n+1}(a)=\min _{f_{1}, \ldots, f_{n} \in E} \max \left\{\|e a e\|: e \in E, e f_{i}=0, i=1,2, \ldots, n\right\} .
\end{aligned}
$$

Proof. We write $s_{n}=s_{n}(a)$ and $a=\Sigma_{n} s_{n} e_{n}$ (see (2.2)). Since for all $e \in$ $E$, $\|$ eae $\|\leqslant\| a \|=s_{1}$ and $s_{1}=\left\|e_{1} a e_{1}\right\|$, it follows that $s_{1}=\max \{\|e a e\|: e \in E\}$. Now let $f_{1}, f_{2}, \ldots, f_{n}$ be any elements in $E$. Put $M_{k}=\left(1-f_{k}\right) A(k=1,2$, $\ldots, n)$. Then by Lemma 3.2, there exists some $h \in E$ such that $h \in M_{1} \cap M_{2}$ $\cap \cdots \cap M_{n} \cap\left(e_{1}+e_{2}+\cdots+e_{n+1}\right) A$. Since $h \in M_{k}$, it follows that $f_{k} h$ $=0(k=1,2, \ldots, n)$. Also $h=\left(e_{1}+e_{2}+\cdots+e_{n+1}\right) h$. Write $h a h=t h$ with $t=\|$ hah\| (Lemma 3.3). Then

$$
\begin{aligned}
\left(t-s_{n+1}\right) h= & h a\left(e_{1}+e_{2}+\cdots+e_{n+1}\right) h-s_{n+1} h \\
= & h\left(s_{1} e_{1}+s_{2} e_{2}+\cdots+s_{n+1} e_{n+1}\right) h \\
& -s_{n+1} h\left(e_{1}+e_{2}+\cdots+e_{n+1}\right) h \\
= & \left(s_{1}-s_{n+1}\right) h e_{1} h+\left(s_{2}-s_{n+1}\right) h e_{2} h+\cdots+\left(s_{1}-s_{n+1}\right) h e_{n} h .
\end{aligned}
$$

Hence it follows from Lemma 3.3 and $[6$, p. 232, Lemma (4.7.4)] that $\left(t-s_{n+1}\right) h$ is positive and so $t=\|h a h\| \geqslant s_{n+1}$. Since $h f_{k}=0$, we have

$$
s_{n+1} \leqslant \max \left\{\|e a e\|: e \in E, e f_{k}=0, k=1,2, \ldots, n\right\} .
$$

Suppose $e \in E$ with $e e_{k}=0(k=1,2, \ldots, n)$. Then

$$
\begin{aligned}
\| \text { eae } \| & =\left\|e\left(\sum_{k=n+1}^{\infty} s_{k} e_{k}\right) e\right\| \leqslant\left\|\sum_{k=n+1}^{\infty} s_{k} e_{k}\right\| \\
& =\sup \left\{\left\|s_{k} e_{k}\right\|: k=n+1, n+2, \ldots\right\}=s_{n+1} .
\end{aligned}
$$


Also $\left\|e_{n+1} a e_{n+1}\right\|=s_{n+1}$. Consequently,

$$
s_{n+1}=\max \left\{\|e a e\|: e \in E, e e_{k}=0, k=1,2, \ldots, n\right\} .
$$

Combining (3.4) and (3.5), we get (3.3) and this completes the proof.

REMARK. Let $H$ be a Hilbert space and $L C(H)$ the algebra of all compact operators on $H$. It is well known that $L C(H)$ is a simple dual $B^{*}$-algebra. For each Hermitian minimal idempotent $e$ in $L C(H)$, we can write $e=(x \otimes x) /(x, x)$ for some nonzero element $x$ in $H$. Then for any Hermitian element $T$ in $L C(H)$, $e T e=(T x, x) e /(x, x)$. Therefore $\|e T e\|=|(T x, x)| /(x, x)$. Let $f=$ $(y \otimes y) /(y, y)$ be any Hermitian minimal idempotent in $L C(H)$. Then it is easy to see that $e f=0$ if and only if $(x, y)=0$. Hence, Theorem 3.4 is a generalization of [2, p. 908, Theorem 3].

The following corollaries are useful in the next section. They are known for the algebra $L C(H)$.

Corollary 3.5. Let a be an element in a dual $B^{*}$-algebra $A$. Then the singular values $s_{n}(a)$ of a are given by

$$
\begin{aligned}
& s_{1}(a)=\max \{\|a e\|: e \in E\} \\
& \quad: \\
& \quad: \\
& s_{n+1}(a)=\min _{f_{1}, \ldots, f_{n} \in E} \max \left\{\|a e\|: e \in E, e f_{i}=0, i=1,2, \ldots, n\right\} .
\end{aligned}
$$

Proof. This follows from Theorem 3.4 and the fact that $\|a e\|^{2}=\|e a * a e\|$ $=\left\|e[a]^{2} e\right\|$.

Corollary 3.6. Let $a \in A$ and $T a$ bounded linear operator on $A$. If $s_{n}(T a)$ are the singular values of $T a$, then we have

$$
s_{n}(T a) \leqslant\|T\| s_{n}(a) \quad(n=1,2, \ldots) .
$$

Proof. Since $\|T a e\| \leqslant\|T\|\|a e\|$, Corollary 3.6 follows easily from Corollary 3.5 .

Corollary 3.7. Let $a$ and $b$ be positive elements in $A$. If $a-b$ is $a$ positive element, then $s_{n}(a) \geqslant s_{n}(b)(n=1,2, \ldots)$.

Proof. Let $e \in E$. Then $e(a-b) e$ is positive. Hence $\|e a e\| \geqslant\|e b e\|$. Therefore by the minimax formula, $s_{n}(a) \geqslant s_{n}(b)$.

4. Some applications of the minimax formula. In this section, by using the minimax formula, we shall generalize some known results for compact operators 
on Hilbert spaces. As before, $A$ will be a dual $B^{*}$-algebra with norm $\|\cdot\|$ and $E$ the set of all Hermitian minimal idempotents of $A$.

Let $R_{n}$ be the set of all elements $x$ in $A$ such that $x=x_{1} f_{1}+x_{2} f_{2}$ $+\cdots+x_{n} f_{n}$, where $x_{1}, x_{2}, \ldots, x_{n} \in A$ and $f_{1}, f_{2}, \ldots, f_{n}$ are mutually orthogonal Hermitian minimal idempotents in $A(n=1,2, \ldots)$. Put $R_{0}=(0)$. Note that $x_{k}(k=1,2, \ldots, n)$ can be zero and so $R_{n-1} \subset R_{n}$.

THeOREM 4.1. Let a be an element in a dual $B^{*}$-algebra $A$. Then for $n=$ $0,1,2, \ldots$, we have

$$
s_{n+1}(a)=\min \left\{\|a-b\|: b \in R_{n}\right\} .
$$

Proof. If $n=0$, then (4.1) reduces to $s_{1}(a)=\|a\|$. Suppose $n \geqslant 1$. Let $b=x_{1} f_{1}+x_{2} f_{2}+\cdots+x_{n} f_{n} \in R_{n}$. If $e \in E$ and $e f_{1}=e f_{2}=\cdots=e f_{n}$ $=0$, then $\|a e\|=\|(a-b) e\| \leqslant\|a-b\|$. Hence it follows from Corollary 3.5 that

$$
s_{n+1}(a) \leqslant\|a-b\| \quad(n=1,2, \ldots) .
$$

Write [a] $=\sum_{k=1}^{\infty} s_{k}(a) e_{k}$ and $a=\Sigma_{k=1}^{\infty} a e_{k}$ (see (2.1) and (2.2)). Put $a_{n}=\Sigma_{k=1}^{n} a e_{k}$. Then $a_{n} \in R_{n}$ and

$$
\begin{aligned}
\left\|a-a_{n}\right\| & =\left\|\sum_{k=n+1}^{\infty} a e_{k}\right\|=\left\|\sum_{k=n+1}^{\infty} e_{k}[a]^{2} e_{k}\right\|^{1 / 2} \\
& =\left\|\sum_{k=n+1}^{\infty} s_{k}^{2}(a) e_{k}\right\|^{1 / 2}=s_{n+1}(a) .
\end{aligned}
$$

Now (4.1) follows immediately from (4.2) and (4.3) and this completes the proof.

Remark. Theorem 4.1 is similar to [3, p. 28, Theorem 2.1].

The following result was obtained by $\mathrm{K}$. Fan for compact operators (see [3, p. 29, Corollary 2.2]).

Corollary 4.2. Let $a, b \in A$. Then the following statements are true for $m, n=1,2, \ldots$.

(i) $s_{m+n-1}(a+b) \leqslant s_{m}(a)+s_{n}(b)$.

(ii) $\left|s_{n}(a)-s_{n}(b)\right| \leqslant\|a-b\|$.

(iii) $s_{m+n-1}(a b) \leqslant s_{m}(a) s_{n}(b)$.

Proof. Let $u \in R_{m-1}$ and $v \in R_{n-1}$ be such that $s_{m}(a)=\|a-u\|$ and $s_{n}(b)=\|b-v\|$ (see the proof of Theorem 4.1).

(i) Since $u+v \in R_{m+n-2}$ (see [1, p. 497]), by Theorem 4.1, we have 


$$
s_{m+n-1}(a+b) \leqslant\|(a+b)-(u+v)\| \leqslant s_{m}(a)+s_{n}(b) .
$$

(ii) This follows immediately from (i).

(iii) Let $w=b-v$ and write $u=x_{1} e_{1}+x_{2} e_{2}+\cdots+x_{m-1} e_{m-1}$, where $e_{1}, e_{2}, \ldots, e_{m-1}$ are mutually orthogonal elements in $E$. We claim that $u w \in R_{m-1}$. In fact, if $e_{i} w \neq 0$, then by $\left[6\right.$, p. 45, Lemma (2.18)], $A e_{i} w$ is a minimal left ideal. Consequently, we can write $A e_{1} w+A e_{2} w+\cdots+A e_{m-1} w$ $=A\left(f_{1}+f_{2}+\cdots+f_{k}\right)$, where $1 \leqslant k \leqslant m-1$ and $f_{1}, f_{2}, \ldots, f_{k}$ are mutually orthogonal elements in $E$ (see [1, p. 497]). Therefore $u w \in R_{m-1}$. Since $(a-u)(b-v)=a b-a v-u w$ and $a v+u w \in R_{m+n-2}$, it follows from Theorem 4.1 that

$$
\begin{aligned}
s_{m+n-1}(a b) & \leqslant\|a b-(a v+u w)\| \leqslant\|a-u\|\|b-v\| \\
& =s_{m}(a) s_{n}(b) .
\end{aligned}
$$

This completes the proof.

By using Corollary 4.2 and the proof of $[3$, p. 32, Theorem 2.3], we have

CoRollary 4.3. Suppose $a, b \in A$ and $r>0$. If $\lim _{n \rightarrow \infty} n^{r} s_{n}(a)=t$ and $\lim _{n \rightarrow \infty} n^{r} s_{n}(b)=0$, then $\lim _{n \rightarrow \infty} n^{r} s_{n}(a+b)=t$.

LEMMA 4.4. Let $a \in A\left(f_{1}+f_{2}+\cdots+f_{n}\right)$, where $f_{1}, f_{2}, \ldots, f_{n}$ are mutually orthogonal Hermitian minimal idempotents in $A$. Then $s_{n+1}(a)=$ $s_{n+2}(a)=\cdots=0$.

Proof. Suppose this is not so. Then we can write $a^{*} a=\sum_{j=1}^{k} s_{j}^{2}(a) e_{j}$ with $n+1 \leqslant k \leqslant \infty$. Since $e_{j} a^{*} a=s_{j}^{2}(a) e_{j}$ and $s_{j}(a) \neq 0$, it follows that $e_{j} \in$ $A\left(f_{1}+f_{2}+\cdots+f_{n}\right)(j=1,2, \ldots, k)$, which is a contradiction (see [1, p. 497]). Hence the lemma is true.

LEMMA 4.5. Let $a \in A$ and $f_{1}, f_{2}, \ldots, f_{k}$ any mutually orthogonal Hermitian minimal idempotents in $A$. Then

$$
\sum_{n=1}^{k}\left\|f_{n} a f_{n}\right\| \leqslant \sum_{n=1}^{k} s_{n}(a) \quad(k=1,2, \ldots) .
$$

Proof. Let $\left\{f_{\beta}\right\}$ be any maximal orthogonal family of Hermitian minimal idempotents containing $\left\{f_{1}, f_{2}, \ldots, f_{k}\right\}$ and $F=f_{1}+f_{2}+\cdots+f_{k}$. Then by Lemma 3.7 in [11] and Lemma 4.4 , we have

$$
\sum_{n=1}^{k}\left\|f_{n} a f_{n}\right\|=\sum_{\beta}\left\|f_{\beta}(a F) f_{\beta}\right\| \leqslant \sum_{n=1}^{\infty} s_{n}(a F)=\sum_{n=1}^{k} s_{n}(a F) .
$$

Since $\|F\|=1$, by Corollary $3.6, s_{n}(a F) \leqslant s_{n}(a)$. Now (4.4) follows easily from (4.5). 
The following lemma is a generalization of a result by K. Fan (see $[3$, p. 48, Lemma 4.2]).

Lemma 4.6. Let $a, b \in A$. Then

$$
\sum_{n=1}^{k} s_{n}(a+b) \leqslant \sum_{n=1}^{k} s_{n}(a)+\sum_{n=1}^{k} s_{n}(b) \quad(k=1,2, \ldots) .
$$

Proof. Write $[a+b]=W^{*}(a+b)$ and $[a+b]=\Sigma_{n} s_{n}(a+b) e_{n}$ (see (2.2) and (2.4)). Then by Lemma 4.5 , we have

$$
\sum_{n=1}^{k} s_{n}(a+b)=\sum_{n=1}^{k}\left\|e_{n}[a+b] e_{n}\right\|=\sum_{n=1}^{k}\left\|e_{n} W^{*}(a+b) e_{n}\right\|
$$

$$
\begin{aligned}
& \leqslant \sum_{n=1}^{k}\left\|e_{n} W^{*} a e_{n}\right\|+\sum_{n=1}^{k}\left\|e_{n} W^{*} b e_{n}\right\| \\
& \leqslant \sum_{n=1}^{k} s_{n}\left(W^{*} a\right)+\sum_{n=1}^{k} s_{n}\left(W^{*} b\right) .
\end{aligned}
$$

Since $\left\|W^{*}\right\|=1,(4.6)$ follows now immediately from (4.7) and Corollary 3.6.

By using Lemma 4.6 and the proof of $[3$, p. 49, Theorem 4.1$]$, we have

THEOREM 4.7. Let $a, b \in A$ and $f(x)(0 \leqslant x<\infty)$ a nondecreasing convex function which vanishes for $x=0$. Then

$$
\sum_{n=1}^{k} f\left(s_{n}(a+b)\right) \leqslant \sum_{n=1}^{k} f\left(s_{n}(a)+s_{n}(b)\right) \quad(k=1,2, \ldots) .
$$

Suppose $a$ is a nonzero element in $A$ with singular values $\left\{s_{n}(a)\right\}$. Define

$$
|a|_{p}=\left(\sum_{n} s_{n}^{p}(a)\right)^{1 / p} \quad(0<p<\infty)
$$

and

$$
|a|_{\infty}=s_{1}(a) \text {. }
$$

For $a=0$, we define $|a|_{p}=0(0<p \leqslant \infty)$. Let $A_{p}=\left\{a \in A:|a|_{p}<\infty\right\}$ $(0<p \leqslant \infty)$. It has been shown that, for $1 \leqslant p \leqslant \infty, A_{p}$ is a dual $A^{*}$-algebra which is a dense two-sided ideal of $A$ and $A_{\infty}=A$ (see [11]). We also obtain that $A_{2}$ is a proper $H^{*}$-algebra with inner product $($,$) such that (x, x)=|x|_{2}^{2}$ and $|a x|_{2} \leqslant\|a\||x|_{2}\left(x \in A_{2}\right)$. Also, for all $x, y \in A_{2}$ and $a \in A$, we have $(a x, y)=\left(x, a^{*} y\right)$ and $(x a, y)=\left(x, y a^{*}\right)$. For each $a \in A$, we define a linear operator $L_{a}$ on $A_{2}$ by

$$
L_{a}(x)=a x \quad\left(x \in A_{2}\right) .
$$


Then $L_{a}$ is a bounded linear operator on $A_{2}$ with $\left\|L_{a}\right\| \leqslant\|a\|$. Hence $L_{a} \in$ $B\left(A_{2}\right)$, the algebra of all bounded linear operators on $A_{2}$. Clearly $\left(L_{a}\right)^{*}=L_{a^{*}}$.

Lemma 4.8. Let $a \in A$. Then $L_{a}$ is a compact operator on $A_{2}$ and $s_{n}(a)$ are the singular values of $L_{a}$.

Proof. Write $a^{*} a=\Sigma_{\alpha} a^{*} a e_{\alpha}=\Sigma_{n} s_{n}(a)^{2} e_{n}$. Let $B$ be the closure of $\left\{L_{a}: a \in A\right\}$ in $B\left(A_{2}\right)$. Then $B$ is a $B^{*}$-algebra. Since $L_{e_{\alpha}} L_{a} L_{e_{\alpha}}=K L_{e_{\alpha}}$, for some constant $k$, it follows that $L_{e_{\alpha}}$ is a Hermitian minimal idempotent in $B$. Let $F \in B$. If $F L_{e_{\alpha}}=0$ for all $\alpha$, then $F L_{e_{\alpha}} x=0$ for all $x$ in $A_{2}$. Hence $F e_{\alpha} x$ $=0$. Since by $\left[10\right.$, p. 442, Theorem 5.2], $x=\Sigma_{\alpha} x e_{\alpha}$ in $1 \cdot I_{2}$, we have $F x=$ $\Sigma_{\alpha} F e_{\alpha} x=0$. Consequently $F=0$ and so $\left\{L_{e_{\alpha}}\right\}$ is a maximal orthogonal family of Hermitian minimal idempotents in $B$. If $M$ is a closed right ideal of $B$ such that $M \supset\left\{L_{e_{\alpha}}\right\}$, then $M \supset\left\{L_{e_{\alpha} b}\right\}$ for all $b \in A$. Since $L_{b}=\Sigma_{\alpha} L_{e_{\alpha} b}$ in $B$, it follows that $L_{b} \in M$ and so $M=B$. Consequently the socle of $B$ is dense in $B$ and so $B$ is a dual $B^{*}$-algebra (see [5, p. 20]). Since $L_{a^{*} a}=\Sigma_{n} s_{n}(a)^{2} L_{e_{n}}$, by the proof of Lemma 2.3 in [11], we see that $s_{n}(a)$ are the singular values of $L_{a}$. Since $L_{a^{*} a}$ is a compact operator on $A_{2}$, so is $L_{a}$ by $[6$, p. 250, Corollary (4.9.3)] and this completes the proof.

The following lemma is a generalization of a result by $\mathbf{A}$. Horn (see $[3, \mathrm{p}$. 48, Lemma 4.2]).

Lemma 4.9. Let $a, b \in A$. Then we have

$$
\prod_{n=1}^{k} s_{n}(a b)=\prod_{n=1}^{k} s_{n}(a) \prod_{n=1}^{k} s_{n}(b) \quad(k=1,2, \ldots)
$$

Proof. Write $[a b]=\Sigma_{n} s_{n}(a b) e_{n}$ and let (, ) be the inner product on $A_{2}$. Since by Lemma 3.1 (iv) in [11], $\left|e_{n}\right|_{2}=\left\|e_{n}\right\|=1$, we have

$$
\left(a b e_{m}, a b e_{n}\right)=\left([a b]^{2} e_{m}, e_{n}\right)= \begin{cases}s_{n}^{2}(a b), & m=n, \\ 0, & m \neq n .\end{cases}
$$

Now it follows easily from Lemma 4.8 and $[4$, p. 375 , Theorem 2] that

$$
\prod_{n=1}^{k} s_{n}^{2}(a b)=\operatorname{det}\left[\left(a b e_{m}, a b e_{n}\right)\right] \leqslant \prod_{n=1}^{k} s_{n}^{2}(a) \prod_{n=1}^{k} s_{n}^{2}(b) .
$$

Therefore (4.9) holds and this completes the proof.

By using Lemma 4.9 and the proof of $[3$, p. 49, Theorem 4.2], we can show that

THEOREM 4.10. Let $a, b \in A$. If the function $f(x)(0 \leqslant x<\infty ; f(0)=0)$ 
becomes convex following the substitution $x=e^{t}(-\infty \leqslant t<\infty)$, then we have

$$
\sum_{n=1}^{k} f\left(s_{n}(a b)\right) \leqslant \sum_{n=1}^{k} f\left(s_{n}(a) s_{n}(b)\right) \quad(k=1,2, \ldots) .
$$

5. Symmetric norming functions and symmetrically-normed ideals. We use the notation in [3, Chapter III]. Let $c_{0}$ be the set of all sequences $\xi=\left\{\xi_{j}\right\}_{1}^{\infty}$ of real numbers which tend to zero and $\hat{c}$ its subset consisting of all sequences with a finite number of nonzero terms. A real function $\Phi(\xi)=\Phi\left(\xi_{1}, \xi_{2}, \ldots\right)$, defined on $\hat{c}$ is called a symmetric norming (s.n.) function (or symmetric gauge function) if it has the following properties:

(I) $\Phi(\xi)>0(\xi \in \hat{c}, \xi \neq 0)$;

(II) for any real constant $k, \Phi(k \xi)=|k| \Phi(\xi)(\xi \in \hat{c})$;

(III) $\Phi(\xi+\eta) \leqslant \Phi(\xi)+\Phi(\eta)(\xi, \eta \in \hat{c})$;

(IV) $\Phi(1,0,0, \ldots)=1$;

(V) $\Phi\left(\xi_{1}, \xi_{2}, \ldots, \xi_{n}, 0,0, \ldots\right)=\left(\left|\xi_{j_{1}}\right|,\left|\xi_{j_{2}}\right|, \ldots,\left|\xi_{j_{n}}\right|, 0,0, \ldots\right)$, where $\xi=\left\{\xi_{j}\right\} \in \hat{c}$ and $\left\{j_{1}, j_{2}, \ldots, j_{n}\right\}$ is any permutation of integers 1,2 , $\ldots, n$. See [3] for some of its properties and an equivalent definition.

As before, $A$ denotes a dual $B^{*}$-algebra with norm $\|\cdot\|$. Let $A^{* *}$ be its second conjugate space with the Arens product. Then it is well known that $A^{* *}$ is a $W^{*}$-algebra and $A$ can be identified as a *-subalgebra of $A^{* *}$ (e.g. see [7] and [10]). The norm on $A^{* *}$ is also denoted by $\|\cdot\|$. By [10, p. 439, Theorem 3.1], $A$ is a two-sided ideal of $A^{* *}$.

We shall make use of the formulas in $\S \S 3$ and 4 to define and study the symmetrically-normed ideals.

Definition. Let $B$ be a subalgebra of $A$ which contains the socle of $A$. A norm $|\cdot|$ on $B$ is called a symmetric norm (or uniform crossnorm) if the following conditions are satisfied:

(i) $|e|=1$ for all Hermitian minimal idempotent $e$ in $A$.

(ii) If $b \in B$ and $a \in A$ such that $s_{j}(a) \leqslant k s_{j}$ (b) for some constant $k$ ( $j=$ $1,2, \ldots)$, then $a \in B$ and $|a| \leqslant k|b|$.

REMARK 1. $B$ is a two-sided ideal of $A^{* *}$. In fact, let $T \in A^{* *}$ and $a \in$ $B$. Since $A$ is a two-sided ideal of $A^{* *}, T a \in A$. By Corollary $3.6, s_{j}(T a) \leqslant$ $\|T\| s_{j}(a)$. Therefore $T a \in B$ by (ii). Similarly $a T \in B$. Hence by (ii), we have $|T a| \leqslant\|T\||a|$ and $|a T| \leqslant\|T\||a|$.

REMARK 2. We have $|\cdot| \geqslant\|\cdot\|$ on $B$. In fact, let $b \in B$ and $e$ be a Hermitian minimal idempotent. Put $a=s_{1}(b) e$. Then $s_{1}(a)=s_{1}(b), s_{2}(a)=$ $s_{3}(a)=\cdots=0$. Hence by (ii), $|a| \leqslant|b|$. Since by (i) $|a|=\left|s_{1}(b) e\right|=s_{1}(b)=$ $\|b\|$, it follows that $\|b\| \leqslant|b|$.

REMARK 3. $\|a e\|=|a e|$ and $\|e a\|=|e a|$ for all $a \in A^{* *}$ and all Hermitian minimal idempotent $e$. In fact, $|a e|=|a e e| \leqslant\|a e\||e|=\|a e\|$. Hence we have 
$|a e|=\|a e\|$. Similarly $|e a|=\|e a\|$. If $A$ is a simple algebra, this property is equivalent to the "cross property" in [8, p. 54, Definition 1 (iv)].

REMARK 4. Since $s_{j}(a)=s_{j}\left(a^{*}\right)=s_{j}([a])=s_{j}\left(\left[a^{*}\right]\right)$ by Lemma 3.1 in [11], it follows that $B$ is a *algebra.

REMARK 5. If $a, b \in B$ with $s_{j}(a)=s_{j}(b)$ for all $j$, then $|a|=|b|$.

Let $S_{A}$ be the socle of $A$. Then $S_{A}$ is a two-sided ideal of $A^{* *}$. In the following result, we shall show that the class of all s.n. functions and the class of all symmetric norms on $S_{A}$ generate each other.

THEOREM 5.1. If $\Phi(\xi)$ is any s.n. function, then the equality

$$
|a|_{\Phi}=\Phi(s(a)) \quad\left(a \in S_{A}, s(a)=\left\{s_{j}(a)\right\}\right)
$$

defines a symmetric norm on $S_{A}$. Conversely, every symmetric norm on $S_{A}$ is obtained in such a manner.

Proof. By Lemma 4.6 and $[3$, p. 75 , Lemma 3.2(v')], we have

$$
\begin{aligned}
|a+b|_{\Phi} & \leqslant \Phi(s(a)+s(b)) \leqslant \Phi(s(a))+\Phi(s(b)) \\
& =|a|_{\Phi}+|b|_{\Phi},
\end{aligned}
$$

for all $a, b$ in $S_{A}$. Since $\Phi(1,0,0, \ldots)=1$, it follows that $|e|_{\Phi}=1$ for all Hermitian minimal idempotents $e$. Property (ii) in Definition easily follows from $\left[3\right.$, p. 71, (3.1)]. Therefore (5.1) defines a symmetric norm on $S_{A}$.

Conversely, let $|\cdot|$ be a symmetric norm on $S_{A}$. Define

$$
\Phi(\xi)=\left|\sum_{j=1}^{n} \xi_{j} e_{j}\right|,
$$

where $\xi=\left\{\xi_{j}\right\}_{j=1}^{n} \in \hat{c}$ and $e_{1}, e_{2}, \ldots, e_{n}$ are mutually orthogonal Hermitian minimal idempotents. It follows easily from Remark 5 that (5.2) is well defined. It is easy to see that $\Phi(\xi)$ is an s.n. function and $|a|_{\Phi}=|a|\left(a \in S_{A}\right)$. This completes the proof.

REMARK. Some argument in the proof of Theorem 5.1 is similar to that of $[8$, p. 65, Theorem 5] and $[3$, p. 78 , Theorem 3.1].

Let $B$ be a subalgebra of $A$ with a symmetric norm $|\cdot|$. If $B$ is complete in $|\cdot|$, then it is called a symmetrically-normed (s.n.) ideal.

REMARK. Since $B$ contains the socle of $A, B$ is an $A^{*}$-algebra which is a dense two-sided ideal of $A$.

Let $\Phi$ be an s.n. function with the natural domain $c_{\Phi}$ (see $[3, \mathrm{p} .80]$ ). For each $a$ in $A$, let $s(a)=\left\{s_{j}(a)\right\}$. Define

$$
A_{\Phi}=\left\{a \in A: s(a) \in c_{\Phi}\right\}
$$


and

$$
|a|_{\Phi}=\Phi(s(a)) \text {. }
$$

REMARK. By using the proof of $[3$, p. 80, Theorem 4.1], we can show that $A_{\Phi}$ is an s.n. ideal with norm $|a|_{\Phi}$.

Two s.n. functions $\Phi(\xi)$ and $\Psi(\xi)$ are said to be equivalent if $\sup \{\Phi(\xi) / \Psi(\xi): \xi \in \hat{c}\}<\infty$ and $\sup \{\Psi(\xi) / \Phi(\xi): \xi \in \hat{c}\}<\infty$

(see [3, p. 76]).

REMARK. It is well known that a semisimple Banach algebra has a unique norm. Therefore two s.n. functions $\Phi(\xi)$ and $\Psi(\xi)$ are equivalent if and only if the s.n. ideal $A_{\Phi}$ and $A_{\Psi}$ coincide elementwise.

Let $\Phi_{\infty}(\xi)$ and $\Phi_{1}(\xi)$ be the minimal and maximal s.n. functions (for definitions, see [3, p. 76]).

REMARK. Since $\|a\|=s_{1}(a)$, it follows that $A_{\Phi}$ coincides with $A$ if and only if $\Phi$ is equivalent to $\Phi_{\infty}$. Since $|a|_{1}=\Sigma_{j=1}^{\infty} s_{j}(a)$, it follows that $A_{\Phi}$ coincides with $A_{1}$ if and only if $\Phi$ is equivalent to $\Phi_{1}$.

As before let $S_{A}$ be the socle of $A$. Then $A_{\Phi}$ contains $S_{A}$. Let $A_{\Phi}^{(0)}$ be the closure of $S_{A}$ in $A_{\Phi}$. We note that $A_{\Phi}^{(0)}$ may not be equal to $A_{\Phi}$ (see [3, p. 87]). Clearly $A_{\Phi}^{(0)}$ is an $A^{*}$-algebra which is a dense two-sided ideal of $A$.

THEOREM 5.2. $A_{\Phi}^{(0)}$ is a dual algebra.

Proof. Let $R_{n}$ be given as in §4. For each $a$ in $A_{\Phi}^{(0)}$, write [a] = $\sum_{j=1}^{\infty} s_{j}(a) e_{j}$ and $a_{n}=\sum_{j=1}^{n} a e_{k}$. Then $a_{n} \in R_{n}$ and by (4.3), $\left\|a-a_{n}\right\|=$ $s_{n+1}(a)$. Therefore by Theorem 4.1, for all $T$ in $R_{r}$, we have

$$
s_{n+1}(a)=\left\|(a+T)-\left(T+a_{n}\right)\right\| \geqslant s_{n+r+1}(a+T) .
$$

Hence by (5.5) and the proof of $[3$, p. 87, Lemma 6.1$]$, we have

$$
\min _{K \in R_{n}}|a-K|_{\Phi}=\left|a-a_{n}\right|_{\Phi}=\Phi\left(s_{n+1}(a), s_{n+2}(a), \ldots\right) .
$$

Since $S_{A}$ is dense in $A_{\Phi}^{(0)}$ and each element of $S_{A}$ belongs to some $R_{n}$, it follows that $a_{n} \rightarrow a$ in $\mid \cdot I_{\Phi}$. Therefore by $[5$, p. 29, Lemma $8(3)], A_{\Phi}^{(0)}$ is a dual $A^{*}$-algebra. This completes the proof.

6. The conjugate space of $A_{\Phi}^{(0)}$. Let $H$ be a Hilbert space with inner product (, ). If $x$ and $y$ are elements in $H$, then $x \otimes y$ will denote the operator on $H$ defined by $(x \otimes y)(h)=(h, y) \cdot x$ for all $h$ in $H$.

LEMMA 6.1. Let $\left\{e_{\alpha}\right\}$ be a net of Hermitian minimal idempotents in $A$ 
such that $\left\{e_{\alpha}\right\}$ converges weakly to some element $e$ in $A^{* *}$. Then $e \in A$.

Proof. Let $\left\{I_{\lambda}\right\}$ be the set of all closed two-sided minimal ideals of $A$. We note first that each $e_{\alpha}$ belongs to some $I_{\lambda}$. Also if $e_{\alpha_{1}} \in I_{\lambda_{1}}$ and $e_{\alpha_{2}} \in I_{\lambda_{2}}$, then $e_{\alpha_{1}} e_{\alpha_{2}}=0$. We divide the proof into two cases.

Case 1. Suppose each $I_{\lambda}$ does not contain any subnet of $\left\{e_{\alpha}\right\}$. Let $e_{\alpha_{0}}$ be an arbitrary element in $\left\{e_{\alpha}\right\}$. Then $e_{\alpha_{0}}$ belongs to some $I_{\lambda_{0}}$. Let $\left\{e_{\beta}\right\}=\left\{e_{\alpha}\right\}$ $\cap I_{\lambda_{0}}$ and $\left\{e_{\gamma}\right\}=\left\{e_{\alpha}\right\}-\left\{e_{\beta}\right\}$. Then $\left\{e_{\gamma}\right\}$ is a subnet of $\left\{e_{\alpha}\right\}$. Since $e_{\gamma} e_{\alpha_{0}}=$ $0, e e_{\alpha_{0}}=0$. Since $e_{\alpha_{0}}$ is arbitrary, it follows that $e e_{\alpha}=0$ for all $\alpha$. Therefore $e^{2}=0$. Since $e=e^{*}$, we have $e=0$. Hence $e \in A$.

Case 2. Suppose there exists some $I_{\lambda}$ which contains a subnet $\left\{e_{\tau}\right\}$ of $\left\{e_{\alpha}\right\}$. It is easy to see that $e \in I_{\lambda}^{* *}$, the second conjugate space of $I_{\lambda}$. Since $I_{\lambda}$ is a simple dual $B^{*}$-algebra, $I_{\lambda}$ has the form $I_{\lambda}=L C\left(H_{\lambda}\right)$ for some Hilbert space $H_{\lambda}$. Also we can identify $L C\left(H_{\lambda}\right)^{* *}$ with $L\left(H_{\lambda}\right)$, the algebra of all continuous linear operators on $H_{\lambda}$. Write $e_{\tau}=x_{\tau} \otimes x_{\tau}$ with $x_{\tau} \in H_{\lambda}$. Since $\left\|x_{\tau}\right\|=1$, we can assume that $\left\{x_{\tau}\right\}$ converges weakly to some $x$ in $H_{\lambda}$. Hence $\left(\left(x_{\tau} \otimes x_{\tau}\right) y, z\right) \rightarrow$ $((x \otimes x) y, z)$ for all $y, z$ in $H$. Since $e_{\tau} \rightarrow e$ weakly in $L C\left(H_{\lambda}\right)^{* *}=L\left(H_{\lambda}\right)$, it follows that $\left(e_{\tau} y, z\right) \rightarrow(e y, z)$ for all $y, z$ in $H$. Therefore $e=x \otimes x \in I_{\lambda} \subset A$. This completes the proof.

The following result is similar to $[3, \mathrm{p} .85$, Theorem 5.2]

LEMMA 6.2. Let $\Phi(\xi)$ be an arbitrary s.n. function not equivalent to the minimal one. If $a$ and $a_{\alpha}$ are positive elements in $A^{* *}$ such that $a_{\alpha} \rightarrow a$ weakly in $A^{* *}, a_{\alpha} \in A_{\Phi}$ and $M=\sup _{a}\left|a_{\alpha}\right|_{\Phi}<\infty$, then $a \in A_{\Phi}$ and $|a|_{\Phi} \leqslant M$.

Proof. Let $a_{\alpha}=\Sigma_{j=1}^{\infty} s_{j}\left(a_{\alpha}\right) e_{j}^{(\alpha)}$ be a spectral representation of $a_{\alpha}$ in $A$ (see (2.2)). For any fixed positive integer $n$, let $b_{\alpha, n}=\Sigma_{j=1}^{n} s_{j}\left(a_{\alpha}\right) e_{j}^{(\alpha)}$. Since $s_{j}\left(a_{\alpha}\right) \leqslant\left|a_{\alpha}\right|_{\Phi} \leqslant M$, there exist subnets $\left\{a_{\beta}\right\}$ and $\left\{e_{j}^{(\beta)}\right\}$ such that $s_{j}\left(a_{\beta}\right) \rightarrow s_{j}$ for some nonnegative number $s_{j}$ and $e_{j}^{(\beta)} \rightarrow e_{j}$ weakly for some $e_{j}$ in $A^{* *}$. By Lemma 6.1, $e_{j} \in A$. Put $b_{n}=\Sigma_{j=1}^{n} s_{j} e_{j}$ and $K_{n}=M / \underbrace{(1,1, \ldots, 1,0, \ldots)}_{n}$
Then $b_{n} \in A$ and

$$
\left\|a_{\alpha}-b_{\alpha, n}\right\|=\left\|\sum_{j=n+1}^{\infty} s_{j}\left(a_{\alpha}\right) e_{j}^{(\alpha)}\right\|=s_{n+1}\left(a_{\alpha}\right) \leqslant K_{n+1} .
$$

Hence for all $f$ in $A^{*}$ with $\|f\| \leqslant 1$, we have

$$
\left|a_{\alpha}(f)-b_{\alpha, n}(f)\right| \leqslant K_{n+1} .(n=1,2, \ldots) .
$$

Since $a_{\beta} \rightarrow a$ and $b_{\beta, n} \rightarrow b_{n}$ weakly in $A^{* *}$, by (6.1) we have 


$$
\begin{aligned}
\left|\left(a-b_{n}\right)(f)\right| & \leqslant\left|\left(a-a_{\beta}\right)(f)\right|+\left|\left(a_{\beta}-b_{\beta, n}\right)(f)\right|+\left|\left(b_{\beta, n}-b_{n}\right)(f)\right| \\
& \leqslant K_{n+1},
\end{aligned}
$$

for all $f$ in $A^{*}$ with $\|f\| \leqslant 1$. Therefore $\left\|a-b_{n}\right\| \leqslant K_{n+1}$. Since $K_{n+1} \rightarrow 0$ as $n \rightarrow \infty$, it follows that $b_{n} \rightarrow a$ and so $a \in A$. Therefore by (2.2), we can write $a=\Sigma_{j=1}^{\infty} s_{j}(a) f_{j}$. Let $t_{j}=\sup _{\alpha} s_{j}\left(a_{\alpha}\right)(j=1,2, \ldots)$. Since $\Phi\left(s_{1}\left(a_{\alpha}\right), s_{2}\left(a_{\alpha}\right), \ldots, s_{n}\left(a_{\alpha}\right), 0,0, \ldots\right) \leqslant M$, it fcllows that

$$
\Phi\left(t_{1}, t_{2}, \ldots, t_{n}, 0,0, \ldots\right) \leqslant M .
$$

Also by Lemma 4.5, we have

$$
\sum_{j=1}^{n}\left\|f_{j} a_{\alpha} f_{j}\right\| \leqslant \sum_{j=1}^{n} s_{j}\left(a_{\alpha}\right) \leqslant \sum_{j=1}^{n} t_{j} .
$$

Since $f_{j} a_{\alpha} f_{j}$ is positive, $f_{j} a_{\alpha} f_{j}=\left\|f_{j} a_{\alpha} f_{j}\right\| f_{j}$. Similarly $f_{j} a f_{j}=\left\|f_{j} a f_{j}\right\| f_{j}$. Since $f_{j} a_{\alpha} f_{j} \rightarrow f_{j} a f_{j}$ weakly in $A^{* *}$, it follows easily that $\left\|f_{j} a_{\alpha} f_{j}\right\| \rightarrow\left\|f_{j} a f_{j}\right\|$. Hence by (6.2), we have

$$
\sum_{j=1}^{n} s_{j}(a)=\sum_{j=1}^{n}\left\|f_{j} a f_{j}\right\| \leqslant \sum_{j=1}^{n} t_{j}
$$

and consequently $\Phi\left(s_{1}(a), s_{2}(a), \ldots, s_{n}(a), 0,0, \ldots\right) \leqslant M$. Therefore $a \in A_{\Phi}$ and this completes the proof.

REMARK. Some argument in the proof of Lemma 6.2 is similar to that given in the proof of $[3$, p. 85, Theorem 5.2].

Let $a \in A_{1}$. Then by Theorem 4.3 in [11], $a=c^{*} b$ for some $b, c$ in $A_{2}$. Define

$$
\operatorname{tr} a=(b, c) \quad\left(a \in A_{1}\right),
$$

where (,) denotes the inner product in $A_{2}$. Let $\left\{f_{\beta}\right\}$ be a maximal orthogonal family of Hermitian minimal idempotents in $A$ and $\lambda_{\beta} f_{\beta}=f_{\beta} a f_{\beta}$. Then by Lemma 4.4 in [11], tr $a$ is well defined, $\operatorname{tr} a=\Sigma_{\beta}\left(a f_{\beta}, f_{\beta}\right)=\Sigma_{\beta} \lambda_{\beta}$ and $|\operatorname{tr} a|$ $\leqslant|a|_{1}$.

Let $\Phi$ be an s.n. function and $\Phi^{*}$ be an s.n. function adjoint to $\Phi$ (see [3,p. 125] and $[8$, p. 69]). By Theorem 4.10 and the proof of $[3$, p. 49, Corollary 4.1], we have

$$
|a x|_{1}=\sum_{j=1}^{\infty} s_{j}(a x) \leqslant \sum_{j=1}^{\infty} s_{j}(a) \sum_{j=1}^{\infty} s_{j}(x) \quad(a, x \in A) .
$$

Also for all $a$ in $A_{\Phi^{*}}$,

$$
|a|_{\Phi}=\max _{0 \neq x \in A}\left(|x|_{\Phi}^{-1} \sum_{j=1}^{\infty} s_{j}(a) s_{j}(x)\right) .
$$

It follows that 


$$
|a x|_{1} \leqslant|a|_{\Phi^{*}}|x|_{\Phi} \quad\left(a \in A_{\Phi^{*}}, x \in A_{\Phi}\right) .
$$

Let $\left(A_{\Phi}^{(0)}\right)^{*}$ be the conjugate space of $A_{\Phi}^{(0)}$. We shall show that $\left(A_{\Phi}^{(0)}\right)^{*}$ can be identified with $A_{\Phi^{*}}$. The following result is a generalization of $[3$, p. 130, Theorem 12.2] .

THEOREM 6.3. Let $\Phi(\xi)$ be an arbitrary s.n. function, not equivalent to the maximal one. Then for each $f$ in $\left(A_{\Phi}^{(0)}\right)^{*}, f$ is of the form

$$
f(x)=\operatorname{tr} a x \quad\left(x \in A_{\Phi}^{(0)}\right),
$$

for some $a$ in $A_{\Phi^{*}}$ and $\|f\|=|a|_{\Phi^{*}}$.

Proof. Let $a \in A_{\Phi^{*}}$ and define $f(x)=\operatorname{tr} a x\left(x \in A_{\Phi}^{(0)}\right)$. Then by (6.4), we have

$$
|f(x)|=|\operatorname{tr} a x| \leqslant|a x|_{1} \leqslant|a|_{\Phi^{*}}|x|_{\Phi} .
$$

Therefore $f \in\left(A_{\Phi}^{(0)}\right)^{*}$ and $\|f\| \leqslant|a|_{\Phi^{*}}$. To show the converse of the inequality, we put $[a]=\sum_{j=1}^{\infty} s_{j}(a) e_{j}$ and $a_{n}=\sum_{j=1}^{n} a e_{j}$. Then

$$
\Phi^{*}\left(s\left(a_{n}\right)\right)=\Phi^{*}\left(s_{1}(a), s_{2}(a), \ldots, s_{n}(a), 0,0, \ldots\right) .
$$

Let $\hat{k}$ be the set of all nonincreasing sequences in $\hat{c}$, each of which consists of nonnegative numbers. Now by the definition of $\Phi^{*}$, we can choose $\xi^{(n)}=$ $\left\{\xi_{j}^{(n)}\right\}_{1}^{n} \in \hat{k}$ such that $\Phi\left(\xi^{(n)}\right)=1$ and $\sum_{j=1}^{n} s_{j}(a) \xi_{j}^{(n)}=\Phi^{*}\left(s\left(a_{n}\right)\right)$. Put $f_{j}=$ $s_{j}(a)^{-2} a e_{j} a^{*}$. Then $\left\{f_{j}\right\}$ are mutually orthogonal Hermitian minimal idempotents and $\left[a^{*}\right]=\sum_{j=1}^{\infty} s_{j}(a) f_{j}$ (see [11]). Let $b_{n}=\sum_{j=1}^{n}\left(\xi_{j}^{(n)} / s_{j}(a)\right) a^{*} f_{j}$. Then $a b_{n}=\sum_{i=1}^{n} \xi_{j}^{n} s_{j}(a) f_{j}$ and so $\operatorname{tr}\left(a b_{n}\right)=\sum_{j=1}^{n} \xi_{j}^{(n)} s_{j}(a)$. Since $b_{n}^{*} b_{n}=$ $\Sigma_{j=1}^{n}\left(\xi_{j}^{(n)}\right)^{2} f_{j}$, we have $s_{j}\left(b_{n}^{*}\right)=\xi_{j}^{(n)}(j=1,2, \ldots, n)$. Therefore

$$
\left|b_{n}\right|_{\Phi}=\Phi\left(s\left(b_{n}\right)\right)=\Phi\left(\xi_{1}^{(n)}, \ldots, \xi_{n}^{(n)}, 0,0, \ldots\right)=1 .
$$

Also

$$
\begin{aligned}
f\left(b_{n}\right) & =\operatorname{tr}\left(a b_{n}\right)=\sum_{j=1}^{n} \xi_{j}^{(n)} s_{j}(a) \\
& =\Phi^{*}\left(s_{1}(a), s_{2}(a), \ldots, s_{n}(a), 0,0, \ldots\right) \\
& =\Phi^{*}\left(s\left(a_{n}\right)\right)=\left|a_{n}\right|_{\Phi^{*}}
\end{aligned}
$$

Since $\left|a_{n}\right|_{\Phi^{*}} \rightarrow|a|_{\Phi^{*}}$ and $\left|b_{n}\right|_{\Phi}=1$, it follows that $\|f\| \geqslant|a|_{\Phi^{*}}$ and so they are equal.

Conversely let $f$ be a nonzero functional in $\left(A_{\Phi}^{(0)}\right)^{*}$. Since $\left.\left|\cdot I_{\Phi} \leqslant\right| \cdot\right|_{1}$ and $S_{A}$ is dense in $A_{\Phi}^{(0)}$, it follows that $f$ is a nonzero functional in $A_{1}^{*}$. Hence by Theorem 3.3 in [12], there exists some $a$ in $A^{* *}$ such that 


$$
f(x)=\operatorname{tr} a x \quad\left(x \in A_{1}\right) .
$$

We show that $a \in A_{\Phi^{*}}$. In fact, let $\left\{e_{\lambda}: \lambda \in \Lambda\right\}$ be a maximal orthogonal family of Hermitian minimal idempotents in $A$ and let $\left\{E_{\gamma}: \gamma \in \Gamma\right\}$ be the direct set of all finite sums $e_{\lambda_{1}}+e_{\lambda_{2}}+\cdots+e_{\lambda_{n}}\left(\lambda_{n} \in \Lambda\right.$ and $\left.n=1,2, \ldots\right)$. Then $\left\|E_{\gamma}\right\|=1$. Define $f_{\gamma}$ on $A_{\Phi}^{(0)}$ by

$$
f_{\gamma}(x)=f\left(x E_{\gamma}\right) \quad\left(x \in A_{\Phi}^{(0)}\right) .
$$

Then

$$
f_{\gamma}(x)=\operatorname{tr}\left(a x E_{\gamma}\right)=\operatorname{tr}\left(E_{\gamma} a x E_{\gamma}\right)=\operatorname{tr}\left(E_{\gamma} a x\right) .
$$

Since $E_{\gamma} a \in A_{\Phi^{*}}$, it follows from the first part of the theorem that $\left|E_{\gamma} a\right|_{\Phi^{*}}=$ $\left\|f_{\gamma}\right\| \leqslant\|f\|$. Since $A^{* *}$ is a $W^{*}$-algebra, by [7, p. 27, Theorem 1.12.1], there exists some $u \in A^{* *}$ with $\|u\|=1$ such that $\left[a^{*}\right]=a u$. Therefore, for all $\gamma$, we have

$$
\left|E_{\gamma}\left[a^{*}\right] E_{\gamma}\right|_{\Phi^{*}}=\left|E_{\gamma} a u E_{\gamma}\right|_{\Phi^{*}} \leqslant\left|E_{\gamma} a\right|_{\Phi^{*}} \leqslant\|f\| .
$$

Hence by the Alaoglu theorem, we can assume that $\left\{E_{\gamma}\left[a^{*}\right] E_{\gamma}\right\}$ converges weakly to some $b$ in $A^{* *}$. Since $\left\{E_{\gamma}\right\}$ converges weakly to the identity in $A^{* *}$, it is easy to see that $\left(E_{\gamma}\left[a^{*}\right] E_{\gamma}\right) e_{\lambda} \rightarrow\left[a^{*}\right] e_{\lambda}$ and $\left(E_{\gamma}\left[a^{*}\right] E_{\gamma}\right) e_{\lambda} \rightarrow b e_{\lambda}$ weakly in $A^{* *}$. Therefore $b e_{\lambda}=\left[a^{*}\right] e_{\lambda}$ for all $\lambda$ and so $b=\left[a^{*}\right]$. Hence $E_{\gamma}\left[a^{*}\right] E_{\gamma} \rightarrow\left[a^{*}\right]$ weakly in $A^{* *}$. Therefore by Lemma $6.2,\left[a^{*}\right] \in A_{\Phi^{*}}$ and so is $a$. This completes the proof.

We remark that some argument in the proof of Theorem 6.3 is similar to that of $[3$, p. 130, Theorem 12.2]

7. Some special symmetrically-normed ideals. Let $\Pi=\left\{\pi_{j}\right\}_{1}^{\infty}$ be an arbitrary binormalizing sequence (see $[3$, p. 141]). Put

$$
A_{\Pi}=\left\{a \in A: \sup _{n}\left[\sum_{j=1}^{n} s_{j}(a) / \sum_{j=1}^{n} \pi_{j}\right]<\infty\right\}
$$

and

$$
A_{\Pi}^{(0)}=\left\{a \in A: \lim _{n \rightarrow \infty}\left[\sum_{j=1}^{n} s_{j}(a) / \sum_{j=1}^{n} \pi_{j}\right]=0\right\} .
$$

THEOREM 7.1. $A_{\Pi}^{(0)}$ and $A_{\Pi}$ are s.n. ideals of $A$ such that $A_{\Pi}^{(0)}$ is a proper subspace of $A_{\Pi}$.

Proof. This follows from the proof of $[3$, p. 141, Theorem 14.1].

Corollary 7.2. $A_{\Pi}$ is a modular annihilator algebra (for definition, see [9]), but not an annihilator algebra. 
Proof. Since $A_{\Pi}$ is a two-sided ideal of $A$ and $A$ is dual, it follows from $\left[9\right.$, p. 830, Theorem 5.2] and $\left[9\right.$, p. 831, Theorem 5.3] that $A_{\Pi}$ is a modular annihilator algebra. Since $S_{A}$ is the socle of $A_{\Pi}$ and $S_{A}$ is not dense in $A_{\Pi}$, it follows that $A_{\Pi}$ is not an annihilator algebra.

Let $\phi_{\pi}$ be defined as in [3, p. 145]. Then $\phi_{\pi}$ is an s.n. function. Let $A_{\pi}$ be the s.n. ideal of $A$ obtained from $\phi_{\pi}$. Then by the proof of [3, p. 149, Theorem 15.2], we have:

THEOREM 73. For the triple of spaces $A_{\Pi}^{(0)}, A_{\pi}$ and $A_{\Pi}$, each space is the conjugate space of the preceding one.

\section{REFERENCES}

1. B. A. Barnes, A generalized Fredholm theory for certain maps in the regular representations of an algebra, Canad. J. Math. 20 (1968), 495-504. MR 38 \#534.

2. N. Dunford and J. T. Schwartz, Linear operators. II: Spectral theory. Self adjoint operators in Hilbert space, Interscience, New York, 1963. MR 32 \#6181.

3. I. C. Gohberg and M. G. Krein, Introduction to the theory of linear nonselfadjoint operators, "Nauka", Moscow, 1965; English transl., Transl. Math. Monographs, vol. 18, Amer. Math. Soc., Providence, R. I., 1969. MR 36 \#3137; 39 \#7447.

4. A. Horn, On the singular values of a product of completely continuous operators, Proc. Nat. Acad. Sci. U.S.A. 36 (1950), 374-375. MR 13, 565.

5. T. Ogasawara and K. Yoshinaga, Weakly completely continuous Banach *algebras, J. Sci. Hiroshima Univ. Ser. A 18 (1954), 15-36. MR 16, 1126.

6. C. E. Rickart, General theory of Banach algebras, University Series in Higher Math., Van Nostrand, Princeton, N. J., 1960. MR 22 \#5903.

7. S. Sakai, $C^{*}$-algebras and $W^{*}$-algebras, Ergebnisse Math. Grenzgebiete, Band 60, Springer-Verlag, Berlin, 1971.

8. R. Schatten, Norm ideals of completely continuous operators, Ergebnisse Math. Grenzgebiete, N.F., Heft 27, Springer-Verlag, Berlin, 1960. MR 22 \#9878.

9. P. K. Wong, Modular annihilator $A$-algebras, Pacific J. Math. 37 (1971), 825-834. MR 46 \#4213.

10. On the Arens products and certain Banach algebras, Trans. Amer. Math. Soc. 180 (1973), 437-448. MR 47 \#7431.

11. - The p-class in a dual $B^{*}$-algebra, Trans. Amer. Math. Soc. 200 (1974), 355-368. MR 50 \#10837.

12. $\longrightarrow$, On certain subalgebras of a dual $B^{*}$-algebra, J. Australian Math. Soc. (to appear).

DEPARTMENT OF MATHEMATICS, SETON HALL UNIVERSITY, SOUTH ORANGE, NEW JERSEY 07079 